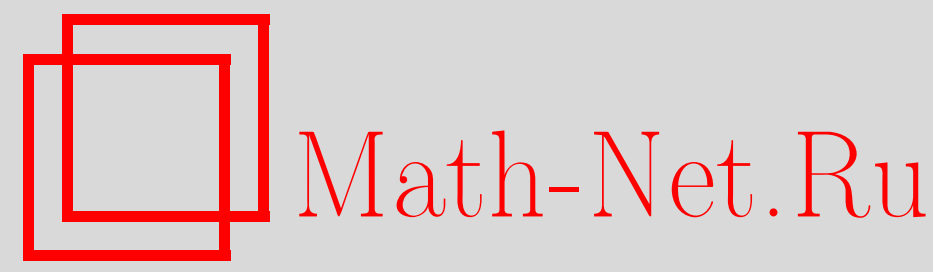

Н. Н. Ганиходжаев, У. А. Розиков, О неупорядоченных фазах некоторых моделей на дереве Кэли, Матем. сб., 1999, том 190, номер 2, 31-42

DOI: https://doi.org/10.4213/sm382

Использование Общероссийского математического портала Math-Net.Ru подразумевает, что вы прочитали и согласны с пользовательским соглашением http://www.mathnet.ru/rus/agreement

Параметры загрузки:

IP : 54.237 .59 .107

26 апреля 2023 г., 15:08:54 
Н. Н. Ганиходжаев, У. А. Розиков

\section{О неупорядоченных фазах некоторых моделей на дереве Кэли}

Рассматриваются модель Изинга с несколькими значениями спина, модель Поттса и неоднородная модель Изинга. Для каждой модели доказаны достаточные условия крайности неупорядоченной фазы.

Библиографйи: 10 названий.

\section{§1. Введение}

Дерево Кэли $\mathscr{T}^{k}$ порядка $k \geqslant 1$ есть бесконечное дерево, т.е. графф без циклов, из каждой вершины которого выходит ровно $k+1$ ребер.

Модель Изинга на дереве Кэли определяется гамильтонианом

$$
H(\sigma)=-\sum_{\langle x, y\rangle} J_{x y} \sigma(x) \sigma(y),
$$

где суммирование ведется по всем парам ближайших соседей $\langle x, y\rangle$ и спиновые переменные $\sigma(x)$ принимают значения \pm 1 .

В [1] установлены необходимые и достаточные условия экстремальности неупорядоченных фаз ферромагнитной модели Изинга. Там же доказано, что вопросы устойчивости неупорядоченных гиббсовских распределений тесно связаны с проблемой фазовых переходов для модели спинового стекла.

Для полноты изложения приведем некоторые обозначения и основные результаты этой работы.

Для ферромагнитной модели Изинга

$$
J_{x y} \equiv J>0
$$

и для модели спинового стекла взаимодействие $J_{x y}$ случайно и

$$
J_{x y}= \pm J, \quad J>0,
$$

с вероятностью $\frac{1}{2}$ независимо относительно любой пары ближайших соседей $\langle x, y\rangle$.

Обе эти модели обладают фазовыми переходами, но значения соответствующих критических температур различны. Положим

$$
\theta=\operatorname{th} \frac{J}{T} .
$$

(C) Н.Н. ГАниходЖАев, У.А. РОЗиков 
Критическое значение $\theta$ для ферромагнитной модели Изинга равно

$$
\theta_{c}^{F}=\frac{1}{k}
$$

и для модели спинового стекла

$$
\theta_{c}^{S G}=\frac{1}{\sqrt{k}}
$$

где $k$ порядок дерева Кэли.

Основным результатом [1] является следующая

ТЕОРема. Неупорядоченное гиббсовское распределение является әкстремальным тогда и только тогда, когда $\theta$ удовлетворяет следующему условию: $0<\theta \leqslant \theta_{c}^{S G}=1 / \sqrt{k}$.

В данной работе изучаются аналогичные вопросы для следующих трех моделей.

1) Неоднородная модель Изинга, гамильтониан которой имеет вид:

$$
H(\sigma)=-\sum_{\langle x, y\rangle} J_{x y} \sigma(x) \sigma(y),
$$

где суммирование ведется по всем парам ближайших соседей $\langle x, y\rangle, J_{x y} \in \mathbb{R}-$ функция, задаюшая взаимодействия соседей $x$ и $y ; \sigma(x) \in\{-1,1\}$ для любого $x \in V$ (где $V$ - множество вершин $\left.\mathscr{T}^{k}\right)$.

2) Модель Изинга с несколькими значениями спина, гамильтониан которой имеет вид:

$$
H(\sigma)=-J \sum_{\langle x, y\rangle} \sigma(x) \sigma(y),
$$

где $J \in \mathbb{R}, \sigma(x) \in\{ \pm 1, \pm 2, \ldots, \pm q\}$ для любого $x \in V$.

3) Модель Поттса, гамильтониан которой имеет вид:

$$
H(\sigma)=-J \sum_{\langle x, y\rangle} \delta_{\sigma(x) \sigma(y)},
$$

где $\delta$ - символ Кронекера, $J \in \mathbb{R}$ и спиновые переменные $\sigma(x)$ принимают значения $\sigma_{1}, \sigma_{2}, \ldots, \sigma_{q} \in \mathbb{R}^{q-1}$ такие, что

$$
\sigma_{i} \sigma_{j}= \begin{cases}1, & \text { если } i=j \\ -\frac{1}{q-1}, & \text { если } i \neq j .\end{cases}
$$

В отличие от результатов [1] в этой работе устанавливаются достаточные условия, при выполнении которых соответствующие неупорядоченные гиббсовские распределения экстремальны, а вопрос о необходимости пока остается открытым. Также пока остается открытым вопрос о взаимосвязи критических температур определенных соответственно для этих трех моделей с проблемой фазовых переходов соответствуюших моделей со случайными взаимодействиями. 
Так как модели, определенные на решетках $\mathbb{Z}^{d}$, точному решению не поддаются, аналогичные вопросы для таких моделей, вообще говоря, не рассматриваются.

В настоящей работе, как и в работах [1]-[8], изучается наиболее простой класс гиббсовских распределений, так назьваемые марковские случайные поля. Для других гиббсовских распределений, более сложной природы, эти вопросы оказываются достаточно сложньми.

В $\S 2$ дается конструкция неупорядоченной фазы. В $\S 3-5$ доказывается, что существуют некоторые значения параметров, для которых неупорядоченная фаза рассматриваемых моделей является экстремальной.

В заключение авторы выражают свою благодарность рецензенту, замечания которого способствовали существенному улучшению содержания и стиля статьи.

\section{§ 2. Конструкция и описание неупорядоченной фазы}

Пусть $L$ - множество ребер $\mathscr{T}^{k}$ и $x^{0} \in V$ - произвольная вершина.

Обозначим

$$
W_{n}=\left\{x \in V: d\left(x^{0}, x\right)=n\right\},
$$

где $d\left(x^{0}, x\right)$ - расстояние в $V$, т.е. для любых $x, y \in V \quad d(x, y)=\min \left\{d: \exists x^{0}=\right.$ $x_{0}, x_{1}, \ldots, x_{d}=x \in V$ такие, что пары $\left\langle x_{0}, x_{1}\right\rangle, \ldots,\left\langle x_{d-1}, x_{d}\right\rangle-$ ближайшие соседи $\}$;

$$
\begin{aligned}
V_{n} & =\left\{x \in V: d\left(x^{0}, x\right) \leqslant n\right\}, \\
L_{n} & =\left\{l=\langle x, y\rangle: x, y \in V_{n}\right\} .
\end{aligned}
$$

Будем говорить, что $x<y$, если путь из $x^{0}$ в $y$ проходит через вершину $x$. Если при этом $x$ и $y$ соседние вершины, то $y$ назьвается "прямым потомком" точки $x$.

Заметим, что $x^{0}$ имеет $k+1$ "прямых потомков", а $x \neq x^{0}$ имеет $k$ "прямых потомков".

Пусть даны гамильтониан $H(\sigma)$ и множество $\Phi$ значений спина.

Для конечного множества $A \subset V$ через $\Omega_{A}=\Phi^{A}$ обозначим пространство конфигураций на множестве $A$. Пусть $h_{x}, x \in V,-$ действительнозначная (в случае модели Поттса векторнозначная) функция.

Для каждого $n$ рассмотрим вероятностную меру $\mu_{n}$ на $\Omega_{V_{n}}$, определенную формулой

$$
\mu_{n}\left(\sigma_{n}\right)=Z_{n}^{-1} \exp \left\{-\beta H\left(\sigma_{n}\right)+\sum_{x \in W_{n}} h_{x} \sigma(x)\right\}
$$

где $\beta=1 / T, \sigma_{n}=\left\{\sigma(x): x \in V_{n}\right\} \in \Omega_{V_{n}}$ и $Z_{n}$ - нормируюший множитель.

Условие согласованности для $\mu_{n}\left(\sigma_{n}\right), n \geqslant 1$, определяется равенством

$$
\sum_{\sigma^{(n)}} \mu_{n}\left(\sigma_{n-1}, \sigma^{(n)}\right)=\mu_{n-1}\left(\sigma_{n-1}\right)
$$

где $\sigma^{(n)}=\left\{\sigma(x): x \in W_{n}\right\}$. 
Пусть $V_{1} \subset V_{2} \subset \cdots, \bigcup_{n=1}^{\infty} V_{n}=V$ и $\left\{\mu_{n}\right\}_{1}^{\infty}$ - последовательность вероятностных мер на $\Omega_{V_{1}}, \Omega_{V_{2}}, \ldots$, обладающая свойством согласованности (5). Тогда в силу теоремы Колмогорова (см., например, [9]) существует, и притом единственная, предельная гиббсовская мера $\mu$ на $\Omega_{V}$ такая, что $\mu\left(\sigma_{n}\right)=\mu_{n}\left(\sigma_{n}\right), n=1,2, \ldots$.

Теорема 1 [2], [3]. Для того чтобъ меры (4) удовлетворяли (5) в случае неоднородной модели Изинга, необходимо и достаточно, чтобъ для $h(x)$, $x \in V$, виполнялось равенство

$$
h_{x}=\sum_{y \in S(x)} f\left(h_{y} ; \theta_{x y}\right)
$$

әде $S(x)$ - множество “прямых потомков" точки $x \in V$,

$$
f(h ; \theta)=\operatorname{arcth}(\theta \operatorname{th} h), \quad \theta_{x y}=\operatorname{th}\left(\beta J_{x y}\right)
$$

Очевидно, что для всех значений параметров $k \geqslant 1, \beta>0, J_{x y} \in \mathbb{R}, x, y \in V$, уравнение (6) имеет решение $h_{x}=0$ для любого $x \in V$.

Обозначим через $\mu_{0}$ гиббсовскую меру, соответствуюшую $\left\{h_{x}=0, x \in V\right\}$. Эта мера называется неупорядоченной фазой неоднородной модели Изинга.

ТЕОРема 2. Для того чтобъ меры (4) удовлетворяли (5) в случае модели Изинга с несколькими значениями спина, необходимо, чтобь для $h_{x}, x \in V$, выполнялось равенство

$$
h_{x}=\frac{1}{q} \sum_{i=1}^{q} \sum_{y \in S(x)} G\left(h_{y} ; J \beta ; q ; i\right)
$$

$2 \partial e$

$$
G(x ; J \beta ; q ; i)=\frac{1}{2 i} \ln \left\{\frac{\operatorname{ch}\left(\frac{q+1}{2}(x+J \beta i)\right) \operatorname{sh}\left(\frac{q}{2}(x+J \beta i)\right) \operatorname{sh}\left(\frac{1}{2}(x-J \beta i)\right)}{\operatorname{ch}\left(\frac{q+1}{2}(x-J \beta i)\right) \operatorname{sh}\left(\frac{q}{2}(x-J \beta i)\right) \operatorname{sh}\left(\frac{1}{2}(x+J \beta i)\right)}\right\} .
$$

ДокаЗАТЕЛьство. Пусть для (4) вьполняется (5), докажем равенство (7). Подставляя (4) в (5), получаем

$$
\begin{gathered}
\frac{Z_{n-1}}{Z_{n}} \sum_{\sigma^{(n)}} \exp \left\{J \beta \sum_{x \in W_{n-1}}\left(\sigma(x) \sum_{y \in S(x)} \sigma(y)\right)+\sum_{x \in W_{n-1}}\left(\sum_{y \in S(x)} h_{y} \sigma(y)\right)\right\} \\
=\exp \left\{\sum_{x \in W_{n-1}} h_{x} \sigma(x)\right\},
\end{gathered}
$$

где $\sigma(x) \in \Phi=\{ \pm 1, \pm 2, \ldots, \pm q\}$. 
Из (8) получим

$$
\frac{Z_{n-1}}{Z_{n}} \prod_{x \in W_{n-1}} \prod_{y \in S(x)}\left(\sum_{\sigma(y)} \exp \left\{J \beta \sigma(x) \sigma(y)+h_{y} \sigma(y)\right\}\right)=\prod_{x \in W_{n-1}} \exp \left\{h_{x} \sigma(x)\right\}
$$

Следовательно,

$$
\prod_{y \in S(x)} \frac{\sum_{\sigma(y)} \exp \left\{J \beta \sigma(x) \sigma(y)+h_{y} \sigma(y)\right\}}{\sum_{\sigma(y)} \exp \left\{-J \beta \sigma(x) \sigma(y)+h_{y} \sigma(y)\right\}}=\exp \left\{2 h_{x} \sigma(x)\right\} .
$$

Положим $\sigma(x)=i, i=1,2, \ldots, q$, тогда из (9) следует

$$
h_{x}=\frac{1}{2 i} \sum_{y \in S(x)} \ln \frac{\sum_{j=1}^{q} \operatorname{ch}\left(\left(J \beta i+h_{y}\right) j\right)}{\sum_{j=1}^{q} \operatorname{ch}\left(\left(-J \beta i+h_{y}\right) j\right)}, \quad i=\overline{1, q} .
$$

Просуммировав (10) по $i$ и использовав формулу

$$
\sum_{i=1}^{n} \operatorname{ch}(i x)=\frac{\operatorname{sh}(n x / 2) \operatorname{ch}((n+1) x / 2)}{\operatorname{sh}(x / 2)}
$$

получим (7). Теорема доказана.

Очевидно, что для всех значений параметров $k, q, J, \beta$ уравнение (7) имеет решение $h_{x}=0, x \in V$, и при этом $\mu_{n}\left(\sigma_{n}\right)$ удовлетворяет условию $(5)$.

Обозначаем через $\mu_{1}$ гиббсовскую меру, соответствующую $\left\{h_{x}=0, x \in V\right\}$. Эта мера назьвается неупорядоченной фазой модели Изинга с несколькими значениями спина.

Теорема 3 [4]. Для того чтобы меры (4) удовлетворяли (5) в случае модели Поттса, необходимо и достаточно, чтобъ для векторов $h_{x}, x \in V$, вицолнялось равенство

$$
h_{x}=\sum_{y \in S(x)} F\left(h_{y} ; \theta ; q\right)
$$

əде $\theta=\exp \left\{\frac{q}{q-1} J \beta\right\}$ и $F: \mathbb{R}^{q-1} \rightarrow \mathbb{R}^{q-1}$ определяется формулой

$$
F_{i}=\ln \left\{\frac{(\theta-1) \exp h_{i}+\sum_{j=1}^{q-1} \exp h_{j}+1}{\sum_{j=1}^{q-1} \exp h_{j}+\theta}\right\}, \quad i=\overline{1, q-1},
$$

$F=\left(F_{1}, F_{2}, \ldots, F_{q-1}\right) u h=\left(h_{1}, h_{2}, \ldots, h_{q-1}\right) \in \mathbb{R}^{q-1}$.

Заметим, что независимо от параметров $k, q, \theta$ совокупность векторов $\left\{h_{x}=(0,0, \ldots, 0) \in \mathbb{R}^{q-1}, x \in V\right\}$ удовлетворяет уравнениям (11).

Обозначим через $\mu_{2}$ меру, соответствуюшую $\left\{h_{x}=(0,0, \ldots, 0) \in \mathbb{R}^{q-1}, x \in V\right\}$. Это мера называется неупорядоченной фазой модели Поттса.

В настоящей работе мы доказываем, что меры $\mu_{0}, \mu_{1}, \mu_{2}$ удовлетворяют следующему свойству. 
Свойство Е. Для любъх $\varepsilon>0, n>0$ и конфигураций $\sigma_{n} \in \Omega_{V_{n}}$ существуют $N>n$ и $A_{N} \subset \Omega_{W_{N}}$ maкuе, что

1) $\mu\left(A_{N}\right)>1-\varepsilon$

2) $\left|\mu\left(\sigma_{n} \mid \sigma^{(N)}\right)-\mu\left(\sigma_{n}\right)\right|<\varepsilon$ для любого $\sigma^{(N)} \in A_{N}$.

Из свойства Е следует, что для типичных граничных условий $\sigma^{(N)}$ условное распределение $\mu\left(\sigma_{n} \mid \sigma^{(N)}\right)$ сходится к безусловному $\mu\left(\sigma_{n}\right)$ при $N \rightarrow \infty$ и $\mu$ является экстремальной мерой (см. [5], [10]).

\section{§3. Экстремальность меры $\mu_{0}$}

Для краткости введем обозначение

$$
\mu_{0}(B)=\mu_{0}\left(B \times \Omega_{V \backslash B}\right), \quad B \subset \Omega_{A} .
$$

Подставляя $h_{x}=0, x \in W_{N}$, в (4), получаем

$$
\mu_{0}\left(\sigma_{N}\right)=Z_{N}^{-1} \exp \left\{-\beta H_{N}\left(\sigma_{N}\right)\right\}
$$

где

$$
H_{N}\left(\sigma_{N}\right)=-\sum_{\langle x, y\rangle \in L_{N}} J_{x y} \sigma(x) \sigma(y)
$$

Обозначим

$$
h_{x}^{(N)}=\beta \sum_{y \in S(x)} J_{x y} \sigma(y), \quad x \in W_{N-1} .
$$

Тогда

$$
\mu_{0}\left(\sigma_{N}\right)=Z_{N}^{-1} \exp \left\{-\beta H_{N-1}\left(\sigma_{N-1}\right)+\sum_{x \in W_{N-1}} h_{x}^{(N)} \sigma(x)\right\} .
$$

Совместное распределение случайных величин $\left\{\sigma(x), x \in V_{N-1}\right\}=\sigma_{N-1}$ и $\left\{h_{x}^{(N)}, x \in W_{N-1}\right\}=h^{(N, N-1)}$ относительно $\mu_{0}$ имеет вид:

$$
\begin{aligned}
& \mu_{0}\left(\sigma_{N-1}, h^{(N, N-1)}\right) \\
& \quad=\widetilde{Z}_{N}^{-1} \exp \left\{-\beta H_{N-1}\left(\sigma_{N-1}\right)+\sum_{x \in W_{N-1}} h_{x}^{(N)} \sigma(x)\right\} \prod_{x \in W_{N-1}} \nu\left(h_{x}^{(N)}\right)
\end{aligned}
$$

где $\nu\left(h_{x}^{(N)}\right)$ - распределение случайной величины $(13)$ при условии, что $\sigma(x)$ независимо принимает значения $-1,1$ с вероятностью $\frac{1}{2}$.

Формула (14) напоминает формулу (4), но здесь значение $h_{x}^{(N)}$ - случайно. Применяя рекуррентные уравнения (6)

$$
h_{x}^{(N)}=\sum_{y \in S(x)} f\left(h_{y}^{(N)} ; \theta_{x y}\right)
$$


определим множество случайных величин

$$
\left\{h_{x}^{(N)}=h_{x}^{(N)}\left(\sigma^{(N)}\right), x \in V_{N-1}\right\}
$$

Тогда совместное распределение случайных величин $\left\{\sigma(x), x \in V_{n}\right\}=\sigma_{n}$ и $\left\{h_{x}^{(N)}, x \in W_{n}\right\}=h^{(N, n)}$ относительно $\mu_{0}$ имеет вид:

$$
\mu_{0}\left(\sigma_{n}, h^{(N, n)}\right)=Z_{N, n}^{-1} \exp \left\{-\beta H_{n}\left(\sigma_{n}\right)+\sum_{x \in W_{n}} h_{x}^{(N)} \sigma(x)\right\} \prod_{x \in W_{n}} \nu_{N-n}\left(h_{x}^{(N)}\right)
$$

где $\nu_{N-n}\left(h_{x}^{(N)}\right)$ - распределение $h_{x}^{(N)}$ для $x \in W_{n}, n<N$. Обозначим

$$
\begin{array}{ll}
a(x)=\sum_{y \in S(x)} \theta_{x y}^{2}, & x \in V, \\
b(x)=\sum_{y \in S(x)}\left|J_{x y}\right|, & x \in V .
\end{array}
$$

Пусть функция $a(x)$ удовлетворяет одному из следующих условий:

УТа) $a(x) \equiv 1$ для любого $x \in V$;

$\mathrm{YIb)} \mathrm{для} \mathrm{любого} x \in V$ и бесконечного пути $\left\{x<x_{1}<x_{2}<\cdots\right\}$ выполняется равенство $\prod_{i=1}^{\infty} a\left(x_{i}\right)=0$,

а функция $b(x)$ удовлетворяет условию

У2) сушествует $M>0$ такое, что $b(x) \leqslant M$ для любого $x \in V$.

Пусть $D h_{x}^{(N)}$ - дисперсия случайной величины $h_{x}^{(N)}=h_{x}^{(N)}\left(\sigma^{(N)}\right)$ относительно меры $\mu_{0}$.

Следующая лемма играет важную роль при доказательстве основного результата этого параграфа.

ЛЕмма 1. Если выполняются одно из условий У1 и условие У2, то

$$
\lim _{N-n \rightarrow \infty} D h_{x}^{(N)}=0, \quad x \in W_{n} .
$$

ДокАЗАТЕльство. Из равенства $(15)$ в силу независимости $h_{x}^{(N)}$ следует, что

$$
D h_{x}^{(N)}=\sum_{y \in S(x)} D f\left(h_{y}^{(N)} ; \theta_{x y}\right), \quad x \in W_{m-1}, \quad y \in W_{m} .
$$

Очевидно, что $|f(t ; \theta)| \leqslant|\theta||t|$.

Из этого неравенства и из (17) следует

$$
D h_{x}^{(N)} \leqslant \sum_{y \in S(x)} \theta_{x y}^{2} D h_{y}^{(N)} \leqslant a(x) D h_{\bar{y}}^{(N)}
$$

где $D h_{\bar{y}}^{(N)}=\max _{y \in S(x)} D h_{y}^{(N)}$. 
Итерируя это неравенство для $x \in W_{n}, y \in W_{N-1}, n \leqslant N-1$, при У1b) получаем

$$
D h_{x}^{(N)} \leqslant\left(\prod_{z: x<z \leqslant y} a(z)\right) D h_{y}^{(N)} .
$$

Из последнего неравенства при У1b) получим (16).

Теперь докажем, что (16) вьполняется и при У1а). В этом случае из (18) получим

$$
D h_{x}^{(N)} \leqslant D h_{\bar{y}}^{(N)}, \quad x \in W_{m-1}, \quad y \in W_{m},
$$

т.е. при возрастании $m$ значения $D h_{x}^{(N)}$ не убывают.

Предположим, что

$$
\lim _{N-n \rightarrow \infty} D h_{x}^{(N)}=\gamma>0, \quad x \in W_{n} .
$$

Тогда для всех $N>n \geqslant 0$

$$
D h_{x}^{(N)} \geqslant \gamma>0, \quad x \in W_{n} .
$$

Пусть $\xi$ - случайная величина и $\varphi(t)-$ ее функция распределения. Для $\varepsilon>0$ введем

$$
D_{\varepsilon} \xi=\int_{|t|>\varepsilon} t^{2} d \varphi(t)
$$

Заметим, что для $|t|>\varepsilon$ сушествует $\delta=\delta(\varepsilon)$ такое, что

$$
|f(h ; \theta)| \leqslant|\theta|(1-\delta)|t| .
$$

Таким образом, для $x \in W_{m-1}, y \in W_{m}$ имеем, что

$$
\begin{aligned}
D h_{x}^{(N)} & \leqslant a(x)\left(\sum_{|t| \leqslant \varepsilon} t^{2} \nu_{N-m}(t)+\sum_{|t|>\varepsilon} t^{2}(1-\delta)^{2} \nu_{N-m}(t)\right) \\
& =\left(D h_{y}^{(N)}-D_{\varepsilon} h_{y}^{(N)}\right)+(1-\delta)^{2} D_{\varepsilon} h_{y}^{(N)} \\
& \leqslant\left(D h_{y}^{(N)}-D_{\varepsilon} h_{y}^{(N)}\right)+(1-\delta) D_{\varepsilon} h_{y}^{(N)} \\
& =D h_{y}^{(N)}-\delta D_{\varepsilon} h_{y}^{(N)}=D h_{y}^{(N)}\left(1-\frac{\delta D_{\varepsilon} h_{y}^{(N)}}{D h_{y}^{(N)}}\right) .
\end{aligned}
$$

Из (13) и У2) следует

$$
\left|h_{y}^{(N)}\right| \leqslant \beta \sum_{z \in S(y)}\left|J_{y z}\right|=\beta b(x) \leqslant \beta M
$$

для любого $y \in V_{N-1}$. Следовательно,

$$
D h_{y}^{(N)} \leqslant \beta^{2} M^{2} .
$$


Таким образом, из (22)

$$
D h_{x}^{(N)}=D h_{y}^{(N)}\left(1-\delta_{0} D_{\varepsilon} h_{y}^{(N)}\right)
$$

где $\delta_{0}=\delta /\left(\beta^{2} M^{2}\right)$. Итерируя эту оценку, получаем

$$
D h_{x}^{(N)} \leqslant D h_{y}^{(N)} \prod_{z: x<z \leqslant y}\left(1-\delta_{0} D_{\varepsilon} h_{z}^{(N)}\right)
$$

В силу (21) из последнего неравенства следует

$$
\lim _{N-n \rightarrow \infty} D_{\varepsilon} h_{x}^{(N)}=0, \quad x \in W_{n}
$$

Так как $\varepsilon>0$ произвольное, то отсюда получим (16) при условии У1а). Лемма доказана.

Теперь докажем свойство Е для $\mu_{0}$ при выполнении условий У1 и У2. Пусть $n>0, \delta>0$ фиксированы. Рассмотрим

$$
\Omega_{N, \delta}=\left\{\sigma^{(N)}:\left|h_{x}^{(N)}\right| \leqslant \delta, x \in W_{N}\right\}
$$

и докажем, что

$$
\lim _{N \rightarrow \infty} \mu_{0}\left(\Omega_{N, \delta}\right)=1
$$

Из (14) получим

$$
\mu_{0}\left(\Omega_{N, \delta}\right)=\frac{\sum_{\left\{\left|h_{x}^{(N)}\right| \leqslant \delta, x \in W_{n}\right\}} Q_{n}\left(\left\{h_{x}^{(N)}, x \in W_{n}\right\}\right)}{\sum_{\left\{h_{x}^{(N)}, x \in W_{n}\right\}} Q_{n}\left(\left\{h_{x}^{(N)}, x \in W_{n}\right\}\right)}
$$

где

$Q_{n}\left(\left\{h_{x}^{(N)}, x \in W_{n}\right\}\right)=\sum_{\sigma_{n}} \exp \left\{-\beta H_{n}\left(\sigma_{n}\right)+\sum_{x \in W_{n}} h_{x}^{(N)} \sigma(x)\right\} \prod_{x \in W_{n}} \nu_{N-n}\left(h_{x}^{(N)}\right)$.

В силу леммы 1

$$
\lim _{N \rightarrow \infty} h_{x}^{(N)}=0, \quad x \in W_{n} .
$$

Таким образом, равенство (23) следует из (24).

Теперь оценим величину $\left|\mu_{0}\left(\sigma_{n} \mid \sigma^{(N)}\right)-\mu_{0}\left(\sigma_{n}\right)\right|$. Из (14) получаем, что

$$
\mu_{0}\left(\sigma_{n} \mid \sigma^{(N)}\right)=\frac{\exp \left\{-\beta H_{n}\left(\sigma_{n}\right)+\sum_{x \in W_{n}} h_{x}^{(N)} \sigma(x)\right\}}{\sum_{\sigma_{n}} \exp \left\{-\beta H_{n}\left(\sigma_{n}\right)+\sum_{x \in W_{n}} h_{x}^{(N)} \sigma(x)\right\}},
$$

где $h_{x}^{(N)}=h_{x}^{(N)}\left(\sigma^{(N)}\right), x \in W_{n}$, выражается через $\sigma^{(N)}$ из формул $(13),(15)$. 
Более того, из (12) следует

$$
\mu_{0}\left(\sigma_{n}\right)=\frac{\exp \left\{-\beta H_{n}\left(\sigma_{n}\right)\right\}}{\sum_{\sigma_{n}} \exp \left\{-\beta H_{n}\left(\sigma_{n}\right)\right\}} .
$$

Так как $n$ фиксировано, то из (25) и (26) следует, что для любого $\varepsilon>0$ сушествует $\delta>0$ такое, что

$$
\left|\mu_{0}\left(\sigma_{n} \mid \sigma^{(N)}\right)-\mu_{0}\left(\sigma_{n}\right)\right|<\varepsilon,
$$

где $\left|h_{x}^{(N)}\right| \leqslant \delta$ для всех $x \in W_{n}$, т.е. для $\sigma^{(N)} \in \Omega_{N, \delta}$.

Следовательно, для данного $\varepsilon>0$ мы можем выбрать такое $\delta>0$, что выполняется (27) для $\sigma^{(N)} \in \Omega_{N, \delta}$, а из (23) следует, что можно выбрать такое $N$, что

$$
\mu_{0}\left(\Omega_{N, \delta}\right)>1-\varepsilon
$$

Таким образом, мы доказали следуюшую теорему

ТЕОРема 4. Если для неоднородной модели Изинга выполняются условия У1) и У2), то неупорядоченная фаза $\mu_{0}$ является әкстремальной.

Заметим, что результат работы [1] получается из теоремы 4 , если предположить, что $\left|J_{x y}\right|=J$ для любых $x, y \in V$.

\section{§4. Экстремальность меры $\mu_{1}$}

Пусть число значений спина $q$ в модели Изинга с несколькими значениями спина имеет вид $q=2^{p}, p=0,1,2, \ldots$, что обусловлено лишь относительной простотой проводимых ниже вычислений.

Ясно, что

$$
\operatorname{sh}\left(2^{p-1} x\right)=2^{p} \operatorname{sh}\left(\frac{x}{2}\right) \prod_{j=-1}^{p-2} \operatorname{ch}\left(2^{j} x\right) .
$$

При $q=2^{p}$, используя (28), из (7) получаем

$$
h_{x}=2^{-p} \sum_{i=1}^{2^{p}} \sum_{y \in S(x)} \frac{1}{i}\left(f\left(\frac{2^{p}+1}{2} h_{y} ; \theta_{i}\right)+\sum_{j=1}^{p} f\left(2^{j-2} h_{y} ; \theta_{i j}\right)\right),
$$

где

$$
f(h ; \theta)=\operatorname{arcth}(\theta \operatorname{th} h), \quad \theta_{i}=\operatorname{th}\left(\frac{2^{p}+1}{2} \beta J i\right), \quad \theta_{i j}=\operatorname{th}\left(2^{j-2} \beta J i\right) .
$$

Обозначим

$$
\begin{gathered}
l_{x}^{(N)}=J \beta \sum_{y \in S(x)} \sigma(y), \quad x \in W_{N-1}, \quad \sigma(y) \in\left\{ \pm 1, \pm 2, \ldots, \pm 2^{p}\right\}, \\
\omega=\omega(k ; \beta ; J ; p)=\sum_{i=1}^{2^{p}} \sum_{j=1}^{p}\left(\frac{1}{i^{2}}\left(\left(\frac{2^{p}+1}{2} \theta_{i}\right)^{2}+\left(2^{j-2} \theta_{i j}\right)^{2}\right)\right) .
\end{gathered}
$$


ЛЕмма 2. Если $0<\omega \leqslant 2^{2 p} / k$, mo

$$
\lim _{N-n \rightarrow \infty} D l_{x}^{(N)}=0, \quad x \in W_{n}
$$

ДокАЗАТЕЛЬСТво аналогично доказательству леммы 1.

Аналогично (как в $\S 3$ ) доказывается, что $\mu_{1}$ удовлетворяет свойству Е. Таким образом, справедлива

Теорема 5. Если $0<\omega \leqslant 2^{2 p} / k$, то неупорядоченная фаза $\mu_{1}$ модели Изинга с несколькими значениями спина является әкстремальной.

Заметим, что результат работы [1] получается из теоремы 5 при $p=0$.

\section{§5. Экстремальность меры $\mu_{2}$}

На $\mathbb{R}^{q-1}$ рассмотрим норму, определенную следующим образом: для $h=\left(h_{1}, h_{2}, \ldots, h_{q-1}\right) \in \mathbb{R}^{q-1}$ положим $\|h\|=\max _{1 \leqslant i \leqslant q-1}\left|h_{i}\right|$.

Нетрудно проверить, что функция $F$ (см. теорему 3 ) удовлетворяет следующим свойствам.

Лемма 3. Для любого $h \in \mathbb{R}^{q-1}$

i) $\|F(h)\| \leqslant \ln \theta$;

ii) $\left|\frac{\partial F_{i}}{\partial h_{j}}\right| \leqslant 2 \ln \theta, i, j=\overline{1, q-1}$;

iii) $\|F(h)\| \leqslant 2(q-1) \ln \theta\|h\|$.

Обозначим

$$
L_{x}^{(N)}=J \beta \sum_{y \in S(x)} \sigma(y), \quad x \in W_{N-1}, \quad \sigma(y) \in\left\{\sigma_{1}, \sigma_{2}, \ldots, \sigma_{q}\right\} \subset \mathbb{R}^{q-1}
$$

Лемма 3 применяется при доказательстве следующей леммы, являющейся аналогом леммы $1 \S 3$.

ЛЕмма 4. Если $\ln \theta \leqslant 1 /(2(q-1) \sqrt{k}), m o$

$$
\lim _{N-n \rightarrow \infty} D\left\|L_{x}^{(N)}\right\|=0, \quad x \in W_{n}
$$

ТЕОрема 6. Если $\ln \theta \leqslant 1 /(2(q-1) \sqrt{k})$, то неупорядоченная фаза $\mu_{2}$ модели Поттса на дереве Кәли является әкстремальной.

ДокАЗАТЕЛЬство аналогично доказательству теоремы 4.

Некоторые другие предельные меры для рассматриваемых моделей, изучены в работах [4], [6]-[8]. 


\section{Список литературы}

1. Bleher P. M. Extremity of the disordered phase in the Ising model on the Bethe lattice // Comm. Math. Phys. 1990. V. 128. P. 411-419.

2. Georgii H. O. Gibbs measures and phase transitions. De Gruyter Studies in Math. V. 9. Berlin: De Gruyter, 1988.

3. Katsura S., Takizawa M. Bethe lattice and the Bethe approximation // Progr. Theoret. Phys. 1974. V. 51. P. 82-98.

4. Ганиходжаев H. Н. О чистых фазах ферромагнитной модели Поттса на решетке Бете // Докл. АН РУз. 1992. №6-7. С. 4-7.

5. Престон К. Гиббсовские состояния на счетных множествах. М.: Мир, 1977.

6. Ганиходжаев Н.Н., Розиков У.А. О периодических гиббсовских распределениях модели Изинга на решетке Бете // Узб. матем. журн. 1995. № 4. С. 8-18.

7. Ганиходжаев Н. Н., Розиков У.А. Описание периодических крайних гиббсовских мер некоторых моделей на дереве Кэли // ТМФ. 1997. Т. 111. № 1. С. 109-117.

8. Розиков У.А. Структуры разбиений на классы смежности группового представления дерева Кэли по нормальным делителям конечного индекса и их применения для описания периодических распределений Гиббса // ТМФ. 1997. Т. 112. №1. С. 170-175.

9. Ширяев А. Н. Вероятность. М.: Наука, 1980.

10. Синай Я.Г. Теория фазовых переходов. М.: Наука, 1980.

Институт математики им. В.И. Романовского АН РУз,

Поступила в редакцию

Ташкент

16.03.1998 